\title{
STARLIKE TREES WITH MAXIMUM DEGREE 4 ARE DETERMINED BY THEIR SIGNLESS LAPLACIAN SPECTRA*
}

\author{
GHOLAM R. OMIDI $^{\dagger}$ AND EBRAHIM VATANDOOST
}

\begin{abstract}
A graph is said to be determined by its signless Laplacian spectrum if there is no other non-isomorphic graph with the same spectrum. In this paper, it is shown that each starlike tree with maximum degree 4 is determined by its signless Laplacian spectrum.
\end{abstract}

Key words. Starlike trees, Spectra of graphs, Cospectral graphs.

AMS subject classifications. $05 \mathrm{C} 50$.

1. Introduction. In this paper, we are only concerned with undirected simple graphs (loops and multiple edges are not allowed). Let $G$ be a graph with $n$ vertices, $m$ edges and the adjacency matrix $A$. We denote the maximum degree of $G$ by $\Delta(G)$. Let $D$ be the diagonal matrix of vertex degrees. The matrices $L=D-A$ and $Q=D+A$ are called the Laplacian matrix and signless Laplacian matrix of $G$, respectively. Since $A, L$ and $Q$ are real symmetric matrices, their eigenvalues are real numbers. So we can assume that $\lambda_{1} \geq \lambda_{2} \geq \cdots \geq \lambda_{n}$ and $\mu_{1} \geq \mu_{2} \geq \cdots \geq \mu_{n}$ are the adjacency and signless Laplacian eigenvalues of $G$, respectively.

Let $M$ be an associated matrix of a graph $G$ (the adjacency matrix, the Laplacian matrix and the signless Laplacian matrix). The multiset of eigenvalues of $M$ is called the $M$ spectrum of $G$. Two graphs are said to be cospectral with respect to $M$ if they have the same $M$ spectrum. A graph is said to be determined ( $D S$ for short) by the $M$ spectrum if there is no other non-isomorphic graph with the same spectrum of $M$. A tree is called starlike if it has exactly one vertex of degree greater than two. We will denote by $S\left(l_{1}, l_{2}, \ldots, l_{r}\right)$ the unique starlike tree such that $S\left(l_{1}, l_{2}, \ldots, l_{r}\right)-v=$ $P_{l_{1}} \cup P_{l_{2}} \cup \ldots \cup P_{l_{r}}$, where $P_{l_{i}}$ is the path on $l_{i}$ vertices $(i=1, \ldots, r)$ and $v$ is the vertex of degree greater than two. A starlike with maximum degree 3 is called a $T$-shape

${ }^{*}$ Received by the editors April 6, 2009. Accepted for publication April 21, 2010. Handling Editor: Richard A. Brualdi.

${ }^{\dagger}$ Department of Mathematical Sciences, Isfahan University of Technology, Isfahan, 84156-83111, Iran (romidi@cc.iut.ac.ir). Also School of Mathematics, Institute for Research in Fundamental Sciences (IPM), P.O. Box 19395-5746, Tehran, Iran). The research of this author was in part supported by a grant from IPM (No.88050012).

${ }^{\ddagger}$ Department of Mathematics, University of Isfahan, Isfahan, 81746-73441, Iran (e.vatandoost@math.ui.ac.ir). The research of this author was partially supported by the Center of Excellence for Mathematics, University of Isfahan. 
and is denoted by $T\left(l_{1}, l_{2}, l_{3}\right)$.

Since the problem of characterization of $D S$ graphs is very difficult, finding any new infinite family of $D S$ graphs is interesting. In [7], it was shown that $T(1,1, n-3)$ and some graphs related to it are determined by their adjacency spectra as well as their Laplacian spectra. In [9], Wang and $\mathrm{Xu}$ proved that $T\left(l_{1}, l_{2}, l_{3}\right)$ is determined by its adjacency spectrum if and only if $\left(l_{1}, l_{2}, l_{3}\right) \neq(l, l, 2 l-2)$ for any integer $l \geq 2$. In [10] they moreover showed that $\mathrm{T}$-shape trees are determined by their Laplacian spectra. Tajbakhsh and Omidi showed that starlike trees are determined by their Laplacian spectra (see [6]). In [5] it has been shown that $T\left(l_{1}, l_{2}, l_{3}\right)$ is determined by its signless Laplacian spectrum if and only if $\left(l_{1}, l_{2}, l_{3}\right) \neq(l, l, 2 l-1)$ for any integer $l \geq 1$. In this paper, we show that each starlike tree with maximum degree 4 is determined by its signless Laplacian spectrum.

2. Preliminaries. First we give some facts that are needed in the next section.

Lemma 2.1. [8](Interlacing) Suppose that $A$ is a symmetric $n \times n$ matrix with eigenvalues $\lambda_{1} \geq \lambda_{2} \geq \cdots \geq \lambda_{n}$. Then the eigenvalues $\mu_{1} \geq \mu_{2} \geq \cdots \geq \mu_{m}$ of a principal submatrix of $A$ of size $m \times m$ satisfy $\lambda_{i} \geq \mu_{i} \geq \lambda_{n-m+i}$ for $i=1, \ldots, m$.

Lemma 2.2. ([8])Let $G$ be a graph. For the adjacency matrix, the Laplacian matrix and the signless Laplacian the following can be obtained from the spectrum.

i) The number of vertices.

ii) The number of edges.

For the adjacency matrix the following follows from the spectrum.

iii) The number of closed walks of any length.

iv) Whether $G$ is bipartite.

LEMma 2.3. [2] The least eigenvalue of the signless Laplacian of a connected graph is equal to 0 if and only if the graph is bipartite. In this case 0 is a simple eigenvalue.

COROLlary 2.4. In any graph (possibly disconnected) the multiplicity of the eigenvalue 0 of the signless Laplacian is equal to the number of bipartite components. The line graph of a starlike tree $S\left(l_{1}, l_{2}, \ldots, l_{r}\right)$ is called the sunlike graph. We will denote this by $K\left(l_{1}, l_{2}, \ldots, l_{r}\right)$.

TheOREM 2.5. [4] If $K\left(l_{1}, l_{2}, \ldots, l_{r}\right)$ and $K\left(l_{1}^{\prime}, l_{2}^{\prime}, \ldots, l_{m}^{\prime}\right)$ are two cospectral sunlike graphs with respect to the adjacency matrix, then they are isomorphic.

Lemma 2.6. [2] Let $G$ be a connected graph and let $H$ be a proper subgraph of $G$. Then $\lambda_{1}(H)<\lambda_{1}(G)$. 
Theorem 2.7. [2] Let $G$ and $H$ be connected graphs and $\{G, H\} \neq\left\{K_{1,3}, K_{3}\right\}$. Then $G$ and $H$ are isomorphic if and only if their line graphs $L(G)$ and $L(H)$ are isomorphic.

LEMma 2.8. [3] Let $G$ be a connected graph that is not isomorphic to $W_{n}$, where $W_{n}$ is a graph obtained from the path $P_{(n-2)}$ (indexed by the natural order of $1,2, \ldots, n-2)$ by adding two pendant edges at vertices 2 and $n-3$. Let $G_{u v}$ be the graph obtained from $G$ by subdividing the edge uv of $G$. If uv lies on an internal path of $G$, then $\lambda_{1}\left(G_{u v}\right) \leq \lambda_{1}(G)$.

Let $n, m, R$ be the number of vertices, the number of edges and the vertex-edge incidence matrix of a graph $G$, respectively. The following relations are well-known:

$$
R R^{T}=A+D, \quad R^{T} R=A_{L}+2 I,
$$

where $D$ is the diagonal matrix of vertex degrees and $A_{L}$ is the adjacency matrix of the line graph $L(G)$ of $G$. Let $P_{L(G)}(\lambda)$ and $Q_{G}(\lambda)$ be characteristic polynomials of $L(G)$ and $G$ with respect to the adjacency and signless Laplacian matrices, respectively. Since non-zero eigenvalues of $R R^{T}$ and $R^{T} R$ are the same, by relations (2.1), we immediately obtain:

$$
P_{L(G)}(\lambda)=(\lambda+2)^{(m-n)} Q_{G}(\lambda+2)
$$

REMARK 2.9. If $m<n$, the matrix $Q$ must have eigenvalue 0 with multiplicity at least $n-m$.

Corollary 2.10. If two graphs $G$ and $G^{\prime}$ are cospectral with respect to the signless Laplacian matrix, then $L(G)$ and $L\left(G^{\prime}\right)$ are cospectral with respect to the adjacency matrix.

The following useful Lemma provides some formulas for calculating the number of closed walks of small lengths.

Lemma 2.11. [5] Let $N_{G}(H)$ be the number of subgraphs of a graph $G$ which are isomorphic to $H$ and let $N_{G}(i)$ be the number of closed walks of length $i$ of $G$. Then:

i) $N_{G}(2)=2 m, N_{G}(3)=6 N_{G}\left(K_{3}\right)$ and $N_{G}(4)=2 m+4 N_{G}\left(P_{3}\right)+8 N_{G}\left(C_{4}\right)$,

ii) $N_{G}(5)=30 N_{G}\left(K_{3}\right)+10 N_{G}\left(C_{5}\right)+10 N_{G}\left(T_{0}\right)$. (see Fig.1)

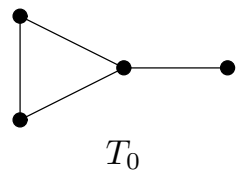

Fig.1 
Lemma 2.12. [1] Let $G$ be a line graph. Then $G$ does not have $T_{i}$ for $i \in\{1,2,3\}$ as an induced subgraph (see Fig.2).

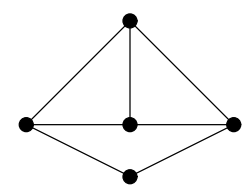

$T_{1}$

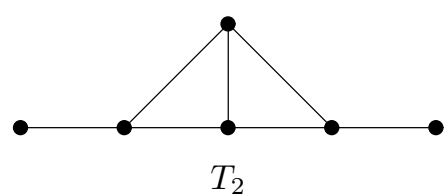

Fig.2

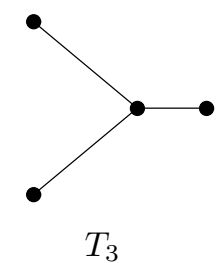

$T_{3}$

3. Main results. Using the previous facts, we show that each non-isomorphic graph $Q$-cospectral to a given starlike tree with maximum degree 4 is either of type $T_{44}$ or a disjoint union of $T_{45}$ with one path (see Fig.7). Finally we show that there is no such graph and so each starlike tree with maximum degree 4 is determined by its signless Laplacian spectrum.

Lemma 3.1. Let $G=K(a, b, c, d)$ with $\min \{a, b, c, d\} \geq 1$. Then:

i) 2 can not be an adjacency eigenvalue of $G$,

ii) If $b=c=d=1$ and $a>1$, then 0 can not be an adjacency eigenvalue of $G$.

Proof.

i) Let 2 be an eigenvalue of $G$ and let $Z \neq 0$ be the eigenvector corresponding to 2 of $G$. Suppose $V(G)=\left\{v_{1}, v_{2}, \cdots, v_{n}\right\}$ be the vertices of $G$ and let $N_{i}=\left\{j \mid v_{i} v_{j} \in\right.$ $E(G)\}$ for $1 \leq i \leq n$. Let $z_{i}$ be the $i$-th entry of $Z$. Since $A Z=2 Z$, for $1 \leq i \leq n$, we have :

$$
\sum_{j \in N_{i}} z_{j}=2 z_{i}
$$

It is easy to see that if $z_{1} z_{a+1} z_{a+b+1} z_{a+b+c+1}=0$, then $Z=0$. Which is not true. So $z_{i} \neq 0$, for $i \in\{1, a+1, a+b+1, a+b+c+1\}$. Using relation (3.1), we have $z_{i}=i z_{1}$ for $1 \leq i \leq a, z_{a+i}=i z_{a+1}$ for $1 \leq i \leq b, z_{a+b+i}=i z_{a+b+1}$ for $1 \leq i \leq c$ and $z_{a+b+c+i}=i z_{a+b+c+1}$ for $1 \leq i \leq d$. Again by relation (3.1), we have $2 z_{a}=z_{a-1}+z_{a+b}+z_{a+b+c}+z_{a+b+c+d}, 2 z_{a+b}=z_{a+b-1}+z_{a}+z_{a+b+c}+z_{a+b+c+d}$, $2 z_{a+b+c}=z_{a+b+c-1}+z_{a+b}+z_{a}+z_{a+b+c+d}$ and $2 z_{a+b+c+d}=z_{a+b+c+d-1}+z_{a}+z_{a+b}+$ $z_{a+b+c}$. So

$$
(2 a-1) z_{1}+(2 b-1) z_{a+1}+(2 c-1) z_{a+b+1}+(2 d-1) z_{a+b+c+1}=0 .
$$

Moreover it is clear that

$$
\begin{gathered}
(2 a+1) z_{1}=(2 b+1) z_{a+1}=(2 c+1) z_{a+b+1}=(2 d+1) z_{a+b+c+1} \\
=a z_{1}+b z_{a+1}+c z_{a+b+1}+d z_{a+b+c+1} .
\end{gathered}
$$


Since $a, b, c$ and $d$ are positive integers, we have $z_{1}=z_{a+1}=z_{a+b+1}=z_{a+b+c+1}=0$, which is not true (see Fig.3).

ii) In the similar way we can prove that 0 can not be the eigenvalue of $G$.

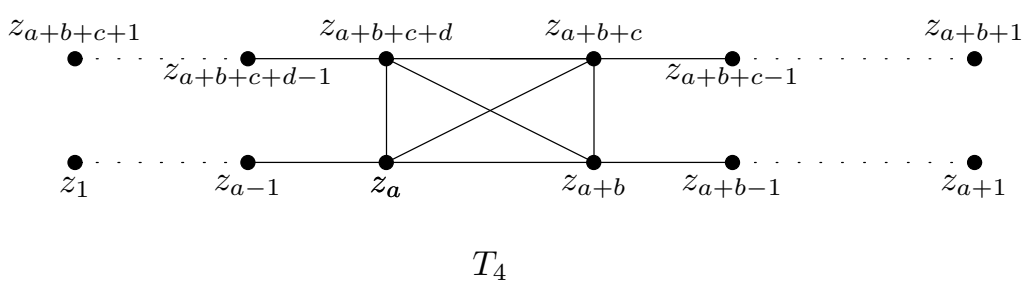

Fig.3

Lemma 3.2. Let $G_{1}=S(a, b, c, d)$ be the starlike tree where $\min \{a, b, c, d\} \geq 1$ and let $G_{2}$ be a cospectral to $G_{1}$ with respect to the signless Laplacian matrix. Let $H_{1}$ and $H_{2}$ be the line graphs of $G_{1}$ and $G_{2}$, respectively. Let $y_{i}$ and $x_{i}$ be the numbers of vertices of degree $i$ of $H_{1}$ and $H_{2}$ respectively. Then:

i) The graph $G_{2}$ has exactly one bipartite component,

ii) $x_{0} \leq 1$,

iii) $\Delta\left(H_{2}\right) \in\{3,4\}$,

iv) $x_{1}=2 x_{4}+x_{3}-2 x_{0}-4$ and $x_{0}+x_{3}+3 x_{4}+2 N_{H_{2}}\left(C_{4}\right)=6+2 y_{4}$,

v) $\lambda_{3}\left(H_{1}\right)<2$.

Proof. i) Since $G_{1}$ is a connected bipartite graph, by Corollary 2.4, $G_{2}$ has exactly one bipartite component.

ii) Each vertex of degree 0 of $H_{2}$ is corresponding to the component $P_{2}$ of $G_{2}$, so by i), $x_{0} \leq 1$.

iii) By Corollary 2.10, two graphs $H_{1}$ and $H_{2}$ are cospectral with respect to the adjacency matrix. By Lemma 2.11 and Lemma 2.2, $N_{H_{1}}\left(K_{3}\right)=N_{H_{2}}\left(K_{3}\right)=4$. So $\Delta\left(H_{2}\right) \geq 2$. If $\Delta\left(H_{2}\right)=2$, then each component of $H_{2}$ is either a path or a cycle. Since each cycle has 2 as an eigenvalue, by Lemma 3.1, $H_{2}$ contains no any cycle as a component. So each component of $H_{2}$ is a path. Hence $N_{H_{2}}\left(K_{3}\right)=0$, which is a contradiction. Now let $\Delta\left(H_{2}\right)=t$ and let $x$ be a vertex of degree $t$ of $H_{2}$. Suppose $e=u v$ be the corresponding edge to $x$ of $G_{2}$. Since $x$ is a vertex of degree $t$, the edge $e=u v$ has $t$ edges of $G_{2}$ as neighborhoods. Let $(\operatorname{deg}(u), \operatorname{deg}(v))=(r, s)$, where $r+s-2=t$. Then $4=N_{H_{2}}\left(K_{3}\right) \geq N_{K_{r}}\left(K_{3}\right)+N_{K_{s}}\left(K_{3}\right)$ and so $r+s \leq 6$. Hence $\Delta\left(H_{2}\right)=t \leq 4$.

iv) Since $H_{1}=L\left(G_{1}\right)=K(a, b, c, d)$, it is clear that $y_{1}=y_{4}, y_{0}=0, y_{3}=4-y_{4}$ and $y_{2}=n-y_{4}-4$. Then by ii) and iii) of Lemma 2.2 , we have $\sum_{i=0}^{4} i^{2} x_{i}+4 N_{H_{2}}\left(C_{4}\right)=$ 
$\sum_{i=0}^{4} i^{2} y_{i}+4 N_{H_{1}}\left(C_{4}\right)$. So

$$
\sum_{i=0}^{4} i^{2} x_{i}+4 N_{H_{2}}\left(C_{4}\right)=4 n+4 y_{4}+24 .
$$

By Lemma 2.2, the number of edges of $H_{2}$ is equal $n+2$, where $n$ is the number of vertices of $H_{1}$. Hence by Lemma 2.2 , we have $\sum_{i=0}^{4} x_{i}=n$ and $\sum_{i=1}^{4} i x_{i}=2 n+4$. By relation (3.2), we have $x_{1}=2 x_{4}+x_{3}-2 x_{0}-4$ and $x_{0}+x_{3}+3 x_{4}+2 N_{H_{2}}\left(C_{4}\right)=6+2 y_{4}$.

v) Let $K$ be a graph obtain by deleting two vertices of degree at least 3 of $H_{1}$. Then each component of $K$ is a path. Since the largest adjacency eigenvalue of each path is less than 2, by Lemma 2.1, we have $\lambda_{3}\left(H_{1}\right) \leq \lambda_{1}(K)<2$.

Lemma 3.3. Let $G_{1}=S(a, b, c, d) \neq K_{1,4}$ and let $G_{2}$ be cospectral graphs with respect to the signless Laplacian matrix. Let $H_{1}$ and $H_{2}$ be the line graphs of $G_{1}$ and $G_{2}$ respectively. If $x$ is the vertex of degree 4 in $H_{2}$, then the induced subgraph of $x$ and its neighborhoods is of type $T_{5}$ or $T_{6}$ (see Fig.4).
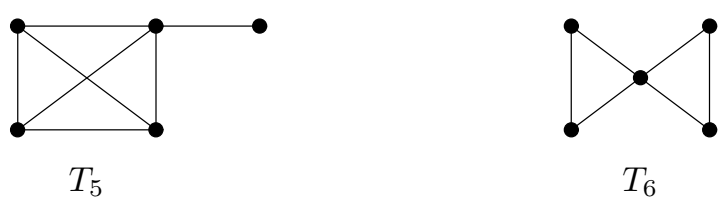

Fig.4

Proof. By Corollary 2.10, two graphs $H_{1}$ and $H_{2}$ are cospectral with respect to the adjacency matrix. So by Lemma 2.2, two graphs $H_{1}$ and $H_{2}$ have the same number of closed walks of length 3 and so by Lemma 2.11, $N_{H_{2}}\left(K_{3}\right)=4$. Let $e=u v$ be corresponding edge of $x$ of $G_{2}$. Since $x$ is a vertex of degree 4, the edge $e=u v$ has 4 edges of $G_{2}$ as neighborhoods. We have the following cases:

Case1: If $(\operatorname{deg}(u), \operatorname{deg}(v)) \in\{(1,5),(5,1)\}$, then $N_{H_{2}}\left(K_{3}\right)>4$. This is impossible.

Case2: If $(\operatorname{deg}(u), \operatorname{deg}(v)) \in\{(2,4),(4,2)\}$, since $N_{H_{2}}\left(K_{3}\right)=4$, then the induced subgraph of $x$ and its neighborhoods is of type $T_{5}$.

Case3: If $(\operatorname{deg}(u), \operatorname{deg}(v))=(3,3)$, then the induced subgraph of $x$ and its neighborhoods is of type $T_{6}, T_{7}$ and $T_{8}$ (see Fig.4 and Fig.5). If the induced subgraph of $x$ and its neighborhoods is of type $T_{7}$, then $x_{0}+x_{3}+3 x_{4}+2 N_{H_{2}}\left(C_{4}\right)>14$. By Lemma 3.2 it is impossible. Now suppose the induced subgraph of $x$ and its neighborhoods be of type $T_{8}$. First suppose $x_{4}=1$. By Lemma 3.1, $H_{2}$ does not have any component of type $C_{5}$. On the other hand by Lemma $3.2, x_{1}=x_{3}-2 x_{0}-2$. Therefore $N_{H_{2}}\left(C_{5}\right)=1$ and so $N_{H_{2}}\left(C_{5}\right)+N_{H_{2}}\left(T_{0}\right) \leq 16$. Moreover $N_{H_{1}}\left(C_{5}\right)=0$ and $N_{H_{1}}\left(T_{0}\right)=12+3 y_{4}$. Since $N_{H_{1}}(5)=N_{H_{2}}(5)$ and $N_{H_{1}}\left(K_{3}\right)=N_{H_{2}}\left(K_{3}\right)=4$, by Lemma 2.11 , we have $y_{4} \in\{0,1\}$. Since $G_{1}=S(a, b, c, d) \neq K_{1,4}$, we have $y_{4}=1$ and so $b=c=d=1$, $a>1$. Therefore by Lemma $3.2,8=6+2 y_{4}=x_{0}+x_{3}+3 x_{4}+2 N_{H_{2}}\left(C_{4}\right) \geq 9$, which 
is impossible.

Now suppose $H_{2}$ has more than one vertex of degree greater than 4 , then $H_{2}$ has $T_{9}, T_{10}$ or $T_{11}$ as a subgraph (see Fig.5). It is easy to see that each graph on 6 vertices with $T_{10}$ as a subgraph has either more than 4 triangles or is not the line graph of any graph. So if $T_{10}$ is a subgraph of $H_{2}$, then it is an induced subgraph. Since $H_{2}=L\left(G_{2}\right)$ and $T_{10}$ is not the line graph of any graph, $H_{2}$ does not have $T_{10}$ as a subgraph. If $H_{2}$ has a subgraph of type $T_{11}$, then by iv) of Lemma 3.2, $2 y_{4}+6=x_{0}+x_{3}+3 x_{4}+2 N_{H_{2}}\left(C_{4}\right) \geq 15$ and so $y_{4} \geq 9 / 2$, which is not true. Now let $H_{2}$ has $T_{9}$ as a subgraph. Since $N_{H_{2}}\left(C_{4}\right) \geq 2$, by iv) of Lemma 3.2, $x_{4} \leq 3$ and so $x_{4} \in\{2,3\}$. If $x_{4}=3$, then by iv) of Lemma $3.2, y_{4}=4$ and $x_{0}+x_{3}=1$. Since $T_{9}$ has 4 vertices of degree greater than $2, H_{2}$ has at least 4 vertices of degree greater than 2. Hence $x_{3}=1$ and $x_{0}=0$. Since $H_{2}$ does not have any cycle as a component, it is easy to see that $H_{2}$ has two components one of them is a path. Using Lemma 2.11, we have $N_{H_{2}}(5)=280$ and $N_{H_{1}}(5)=360$, which is not true. If $x_{4}=2$, since $H_{2}$ has at least 4 vertices of degree greater than $2, x_{3} \geq 2$. By iv) of Lemma 3.2, $x_{0}+x_{3} \leq 4$. If $x_{3}=2$, then $H_{2}$ has two components, one of them is $T_{9}$ and another is a path. By iv) of Lemma 3.2, $y_{4}=3$ and using Lemma 2.11, we have $N_{H_{2}}(5)=270$ and $N_{H_{1}}(5)=330$, which is not true. Now let $H_{2}$ has 3 or 4 vertices of degree 3 . Using Lemma 2.11, we have $N_{H_{2}}(5) \in\{280,290\}$ and $N_{H_{1}}(5)=240+30 y_{4}$ is a multiple of 30. Thus $N_{H_{1}}(5) \neq N_{H_{2}}(5)$, which is not true.

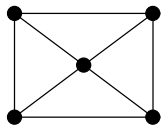

$T_{7}$

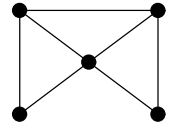

$T_{8}$

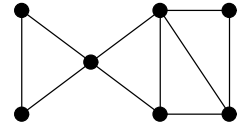

$T_{9}$

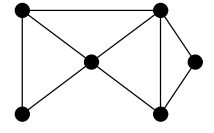

$T_{10}$

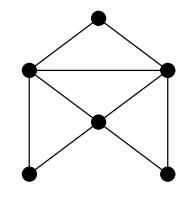

$T_{11}$

Fig.5

Let $H_{1}=K(a, b, c, d)$ and $H_{2}$ be non-isomorphic cospectral graphs with respect to the adjacency matrix. Let $N$ be the number of cycles of $H_{2}$ where the induced subgraph obtained by its vertices contains no any triangle as a subgraph. Again let $x_{i}$ be the number of vertices of degree $i$ of $H_{2}$. We have the following useful lemma.

Lemma 3.4. Let $\mathrm{H}_{2}$ does not have $K_{4}$ as a subgraph. Then:

i) $H_{2}$ contains exactly one $T_{i}$ as a subgraph for $12 \leq i \leq 43$ where different components of $\mathrm{T}_{i}$ lie in the different components of $\mathrm{H}_{2}$ (see Fig.6).

Also if $\mathrm{H}_{2}$ contains $\mathrm{T}_{l}$ as a subgraph for $12 \leq l \leq 40$, then

ii) If $\mathrm{H}_{2}$ contains no path as a component or $x_{0}=1$, then $x_{1}+2 \mathrm{~N}=x_{3}-s$,

iii) If $x_{0}=0$ and $H_{2}$ contains exactly one path as a component, then $x_{1}+2 N=$ $x_{3}-r$. 
Where,

$$
(s, r)= \begin{cases}(6,4) & 12 \leq i \leq 16 \\ (4,2) & 17 \leq i \leq 28 \\ (2,0) & 29 \leq i \leq 37 \\ (0,-2) & 38 \leq i \leq 40\end{cases}
$$
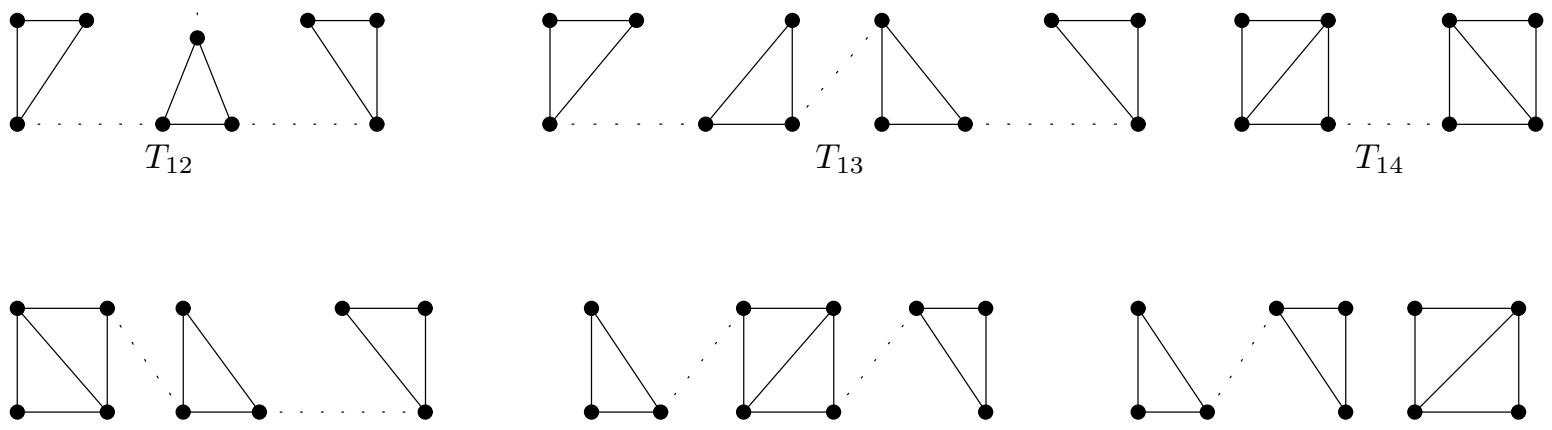

$T_{15}$

$T_{16}$

$T_{17}$

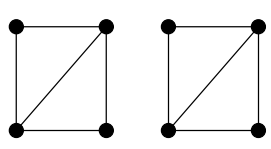

$T_{18}$
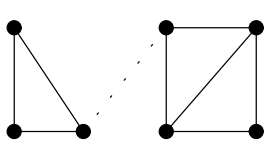

$T_{19}$

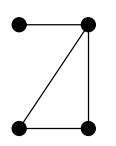

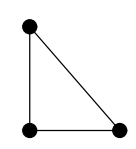

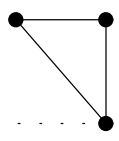

$T_{20}$
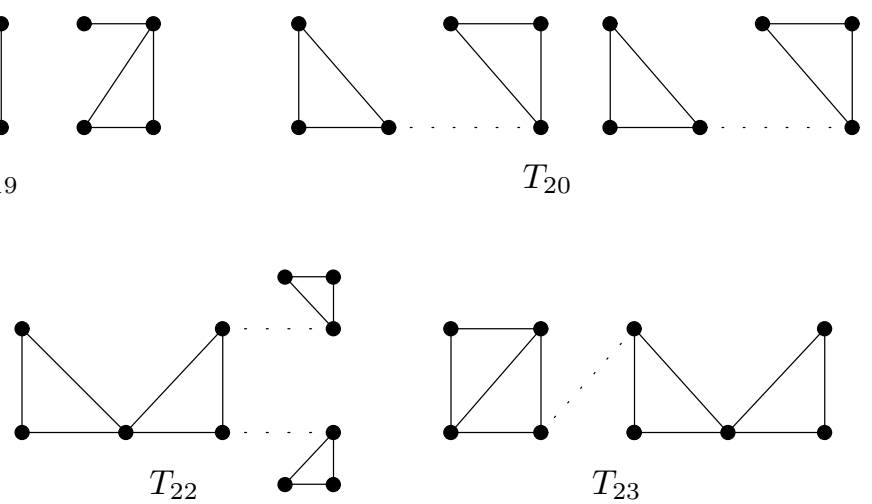

$T_{23}$
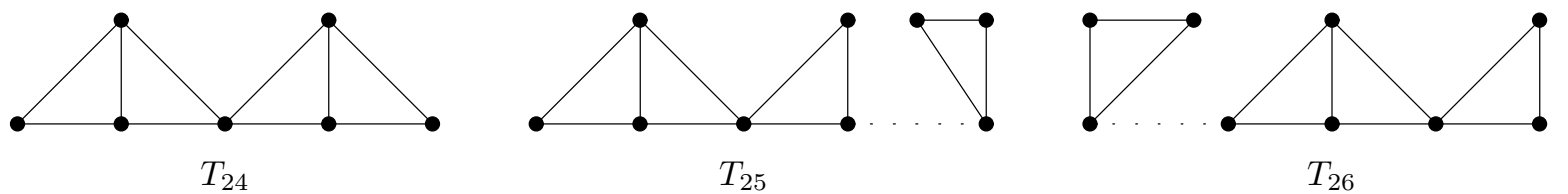

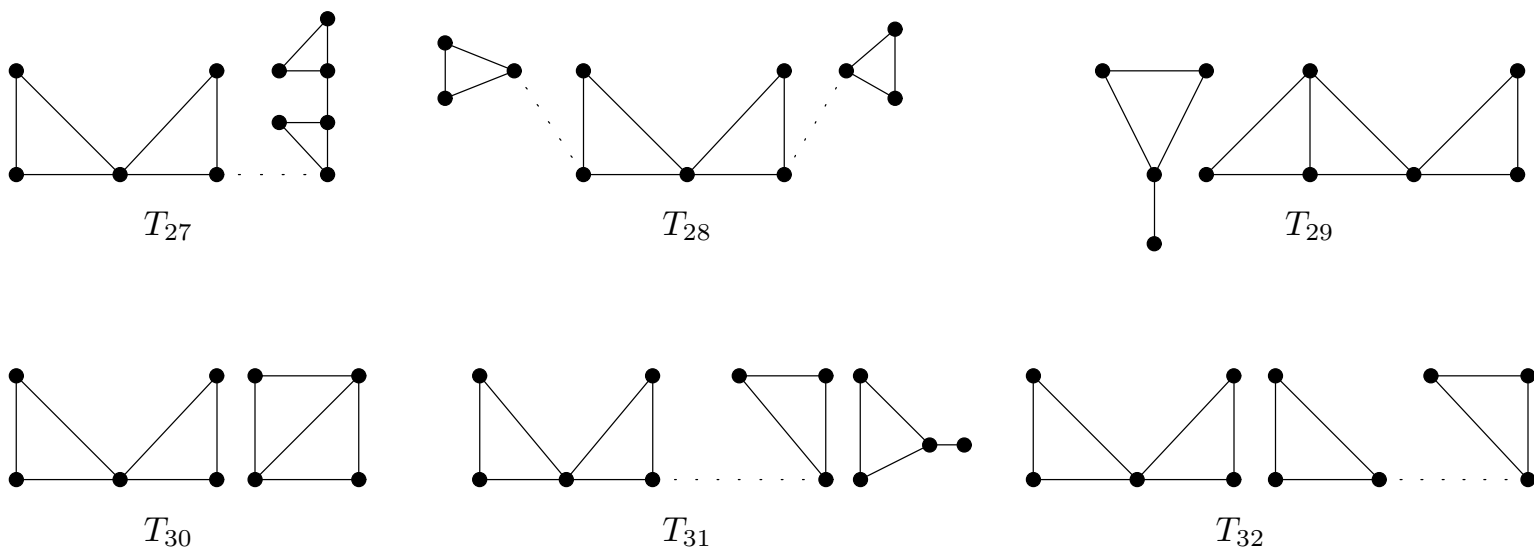

$T_{31}$

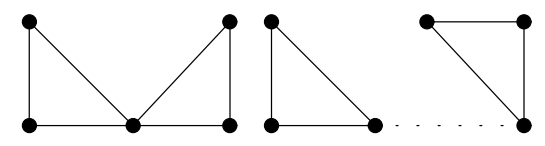

$T_{32}$
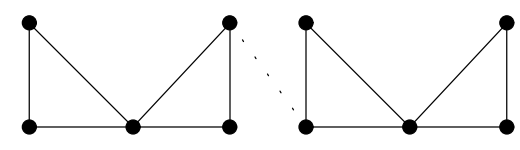

$T_{33}$

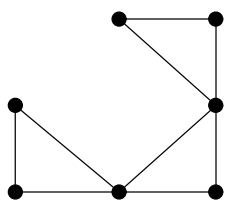

$T_{36}$
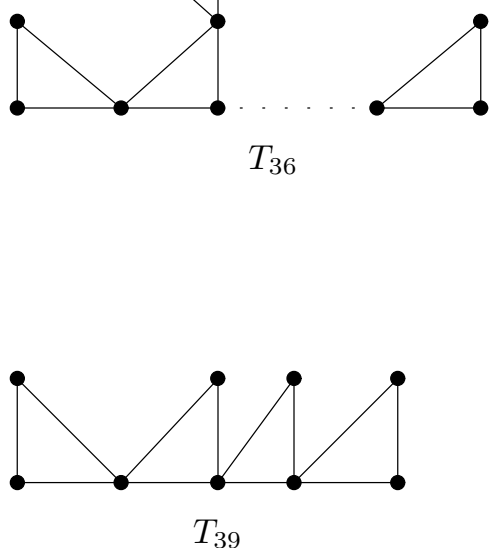

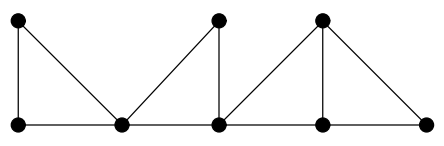

$T_{34}$

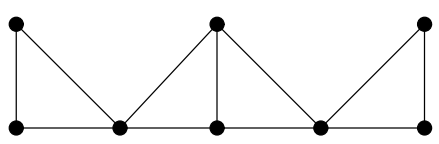

$T_{37}$

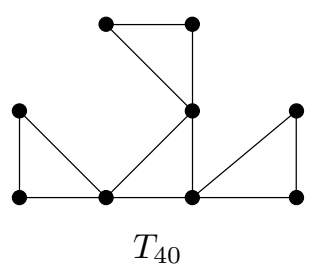

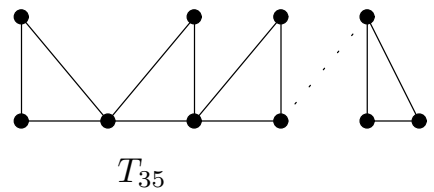
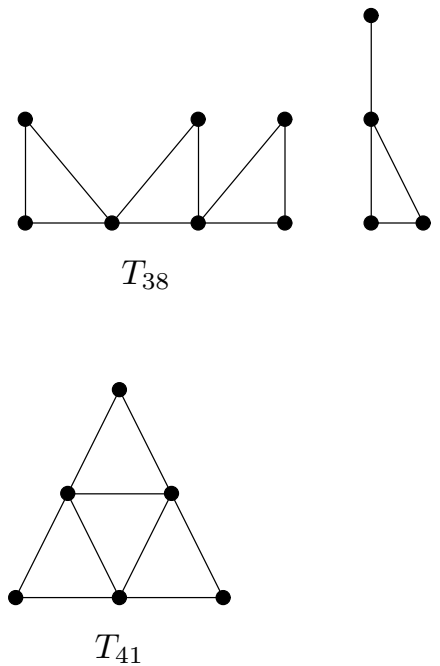


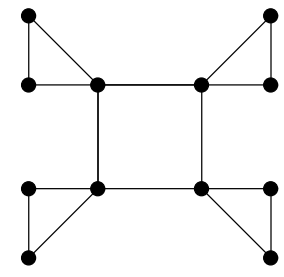

$T_{42}$

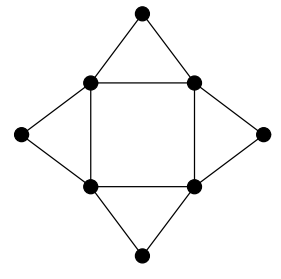

$T_{43}$

Fig.6

Proof. i) Since $H_{1}$ and $H_{2}$ are cospectral with respect to the adjacency matrix, by Lemma $2.2, N_{H_{1}}(3)=N_{H_{2}}(3)$ and so by Lemma 2.11 , we have $N_{H_{2}}\left(K_{3}\right)=4$. By Lemma 3.2, $\lambda_{3}\left(H_{2}\right)<2$ and so the number of non-tree components of $H_{2}$ is at most 2. Moreover by Lemma 3.1, $H_{2}$ does not have any cycle as a component. On the other hand by Lemma 2.12, $H_{2}$ does not have $K_{1,3}$ as an induced subgraph and so each non-tree component of $H_{2}$ has $K_{3}$ as a subgraph. Let $K$ be a disjoint union of non-tree components of $H_{2}$. By Lemma 3.2, $\Delta\left(H_{2}\right) \in\{3,4\}$. Note that by Lemma 3.3 , each vertex of degree 4 of $H_{2}$ is a vertex of $K$. If $\Delta\left(H_{2}\right)=3$, then $K$ has exactly one $T_{i}$ for $12 \leq i \leq 21$ as a subgraph. Now let $\Delta\left(H_{2}\right)=4$. If $x_{4}=1$, then $K$ has exactly one $T_{i}$ for $22 \leq i \leq 32$ as a subgraph. If $x_{4}=2$, then $K$ has exactly one $T_{i}$ for $33 \leq i \leq 38$ as a subgraph. If $x_{4}=3$, then $K$ has exactly one $T_{i}$ for $i \in\{39,40,41\}$ as a subgraph. If $x_{4}=4$, then $K$ has exactly one $T_{i}$ for $i \in\{42,43\}$ as a subgraph.

ii, iii) Since $H_{2}$ does not have $K_{1,3}$ as an induced subgraph and $N_{H_{2}}\left(K_{3}\right)=4$, each vertex of degree 3 of $H_{2}$ is a vertex of subgraph $T_{l}$. It is clear that the number of vertices with degree 2 of $T_{l}$ where their degrees are 3 in $H_{2}$ is equal to $2 N$ plus the number of vertices with degree 1 of $V\left(H_{2}\right) \backslash V\left(T_{l}\right)$. Let $z_{i}$ be the number of vertices of degree $i$ of $T_{l}$. So $x_{3}-z_{3}=2 N+x_{1}-z_{1}$. If $s=z_{3}-z_{1}$, where $H_{2}$ contains no any path as a component or $x_{0}=1$, then $x_{1}+2 N=x_{3}-s$. If $r=z_{3}-z_{1}$, where $x_{0}=0$ and $H_{2}$ contains exactly one path as a component, then $x_{1}+2 N=x_{3}-r$. $\square$

Lemma 3.5. Let $G_{1}=S(a, b, c, d) \neq K_{1,4}$ and $G_{2}$ be cospectral graphs with respect to the signless Laplacian matrix. Let $H_{2}$ be the line graph of $G_{2}$. Then $\Delta\left(H_{2}\right)=4$.

Proof. By Corollary 2.10, the graphs $H_{1}=L\left(G_{1}\right)$ and $H_{2}$ are cospectral with respect to the adjacency matrix. So by Lemma 2.2 and Lemma $2.11, N_{H_{2}}\left(K_{3}\right)=4$. Let $x_{i}$ be the number of vertices of degree $i$ of $H_{2}$, by Lemma 3.2, it is sufficient to show that $\Delta\left(H_{2}\right) \neq 3$. Let $\Delta\left(H_{2}\right)=3$. By Lemma $3.1, H_{2}$ does not have any cycle as a component. If $H_{2}$ has $K_{4}$ as a subgraph, then since $\Delta\left(H_{2}\right)=3, K_{4}$ is the component of $H_{2}$. Since $L\left(G_{2}\right)=H_{2}$ and $N_{H_{2}}\left(K_{3}\right)=N_{K_{4}}\left(K_{3}\right)=4$, all other components of $H_{2}$ are trees. By Lemma 3.2, $G_{2}$ has exactly one bipartite component. Hence $H_{2}=K_{4}$ and so $G_{2}=K_{1,4}$ and by Theorem $2.2, G_{1}=K_{1,4}$. Which is impossible. So $H_{2}$ does 
not have $K_{4}$ as a subgraph. Since $N_{H_{2}}\left(K_{3}\right)=4$ and $x_{4}=0, H_{2}$ has a subgraph of type $T_{i}$ for $12 \leq i \leq 21$ (see Fig.6).

Step1: Let $12 \leq i \leq 16$. If $x_{0}=1$, then by Lemma $3.2, x_{1}=x_{3}-6$ and by Lemma $3.4, x_{1}+2 N=x_{3}-6$. So $N=0$. If $x_{0}=0$ and $H_{2}$ contains exactly one path as a component, then by Lemma 3.2, $x_{1}=x_{3}-4$ and by Lemma 3.4, $x_{1}+2 N=x_{3}-4$. Hence $N=0$. So by Lemma 3.2, for $i \in\{12,13\}$, we have $x_{3}=5+2 y_{4}$ or $x_{3}=6+2 y_{4}$. Hence by Lemma $2.11, N_{H_{2}}(5)=170+20 y_{4}$ or $N_{H_{2}}(5)=180+20 y_{4}$. On the other hand $N_{H_{1}}(5)=240+30 y_{4}$. However $N_{H_{1}}(5) \neq N_{H_{2}}(5)$. Which is a contradiction to this fact $H_{1}$ and $H_{2}$ are cospectral with respect to adjacency matrix. For $i=14$ , we have $x_{3}=1+2 y_{4}$ or $x_{3}=2+2 y_{4}$. By Lemma 2.11, $N_{H_{2}}(5)=170+20 y_{4}$ or $N_{H_{2}}(5)=180+20 y_{4}$. On the other hand $N_{H_{1}}(5)=240+30 y_{4}$. So $N_{H_{1}}(5) \neq$ $N_{H_{2}}(5)$. Which is a contradiction to this fact $H_{1}$ and $H_{2}$ are cospectral with respect to adjacency matrix. For $i=15$, we have $x_{3}=3+2 y_{4}$ or $x_{3}=4+2 y_{4}$. By Lemma 2.11, $N_{H_{2}}(5)=170+20 y_{4}$ or $N_{H_{2}}(5)=180+20 y_{4}$. On the other hand $N_{H_{1}}(5)=240+30 y_{4}$. So $N_{H_{1}}(5) \neq N_{H_{2}}(5)$. Which is a contradiction to this fact $H_{1}$ and $H_{2}$ are cospectral with respect to adjacency matrix. If $H_{2}$ contains no any path as a component, then by Lemma $3.2, x_{1}=x_{3}-4$ and by Lemma $3.4, x_{1}+2 N=x_{3}-6$. Hence $N=-1$, which is impossible. It is easy to see that if $H_{2}$ has $T_{16}$ as a subgraph, then it has either $T_{2}$ or $T_{3}$ as an induced subgraph. Since $H_{2}=L\left(G_{2}\right)$ by Lemma 2.12, which is impossible.

Step2: Let $17 \leq i \leq 21$. First let $i \in\{17,19\}$. If $x_{0}=1$, then by Lemma 3.2, $x_{1}=x_{3}-6$ and by Lemma 3.4, $x_{1}+2 N=x_{3}-4$. Also if $x_{0}=0$ and $H_{2}$ contains exactly one path as a component, then $x_{1}+2 N=x_{3}-2$ and $x_{1}=x_{3}-4$. Any way we have $N=1$. Thus $N_{H_{2}}\left(C_{4}\right)=1$ or 2 . If $N_{H_{2}}\left(C_{4}\right)=1$, then by Lemma 3.2 , we have $x_{3}=3+2 y_{4}$ or $x_{3}=4+2 y_{4}$. By Lemma 2.11, $N_{H_{2}}(5)=170+20 y_{4}$ or $N_{H_{2}}(5)=180+20 y_{4}$. If $N_{H_{2}}\left(C_{4}\right)=2$, then $x_{3}=1+2 y_{4}$ or $x_{3}=2+2 y_{4}$. So $N_{H_{2}}(5)=150+20 y_{4}$ or $N_{H_{2}}(5)=160+20 y_{4}$. Moreover $N_{H_{1}}(5)=240+30 y_{4}$. However, this is a contradiction to this fact $H_{1}$ and $H_{2}$ are cospectral with respect to adjacency matrix. If $H_{2}$ contains no any path as a component, then by Lemma $3.2, x_{1}=x_{3}-4$ and by Lemma 3.4, $x_{1}+2 N=x_{3}-4$. Therefor $N=0$ and so $N_{H_{2}}\left(C_{4}\right)=1$. By Lemma $3.2, x_{3}=4+2 y_{4}$. So $N_{H_{2}}(5)=180+20 y_{4}<240+30 y_{4}$. Which is impossible. Let $i=18$. If $x_{0}=1$, then by Lemma 3.2, $x_{1}=x_{3}-6$ and by Lemma 3.4, $x_{1}+2 N=x_{3}-4$. If $x_{0}=0$ and $H_{2}$ contains exactly one path as a component, then $x_{1}+2 N=x_{3}-2$ and $x_{1}=x_{3}-4$, respectively. Any way we have $N=1$. Since by Lemma $2.12, H_{2}$ does not have $T_{1}$ as an induced subgraph, we have $N_{H_{2}}\left(C_{4}\right)=2$. So by Lemma $3.2, x_{3}=1+2 y_{4}$ or $x_{3}=2+2 y_{4}$. So $N_{H_{2}}(5)=170+20 y_{4}$ or $N_{H_{2}}(5)=180+20 y_{4}$. However, $N_{H_{2}}(5)<240+30 y_{4}$ this is not true. If $H_{2}$ contains no any path as a component, then $x_{1}+2 N=x_{3}-4$ and $x_{1}=x_{3}-4$. Hence $N=0$ and so $N_{H_{2}}\left(C_{4}\right)=2$. By Lemma $3.2, x_{3}=2+2 y_{4}$. So $N_{H_{2}}(5)=180+20 y_{4}<N_{H_{1}}(5)$, which is a contradiction to this fact $H_{1}$ and $H_{2}$ are cospectral with respect to adjacency matrix. 
Now let $i \in\{20,21\}$. If $x_{0}=1$, then by Lemma 3.2, $x_{1}=x_{3}-6$ and by Lemma 3.4, $x_{1}+2 N=x_{3}-4$. If $x_{0}=0$ and $H_{2}$ contains exactly one path as a component, then $x_{1}+2 N=x_{3}-2$ and $x_{1}=x_{3}-4$, respectively. Therefore $N=1$ and so $G_{2}$ has more than one bipartite component which is impossible. If $H_{2}$ contains no any path as a component, then by Lemma 3.2, $x_{1}=x_{3}-4$ and by Lemma 3.4, $x_{1}+2 N=x_{3}-4$. Hence $N=0$ and so $G_{2}$ has more than one bipartite component, which is false.

In the following theorem by using the previous facts we show that only graphs of type $T_{44}$ and disjoint union of $T_{45}$ with one path can be cospectral to a given starlike tree with maximum degree 4 with respect to the signless Laplacian spectrum (see Fig.7).

Theorem 3.6. Let $G_{1}=S(a, b, c, d)$ where $d \geq c \geq b \geq a \geq 1$ and let $G_{2}$ be cospectral to $G_{1}$ with respect to the signless Laplacian matrix. Then:

i) If $a=b=1$, then $G_{1}$ and $G_{2}$ are isomorphic,

ii) If $a=1, b>1$, then $G_{2}$ is either isomorphic to $G_{1}$ or is of type $T_{44}$,

iii) If $a>1$, then $G_{2}$ is either isomorphic to $G_{1}$ or it has two components, one of them is path and another is of type $T_{45}$ (see Fig.7).
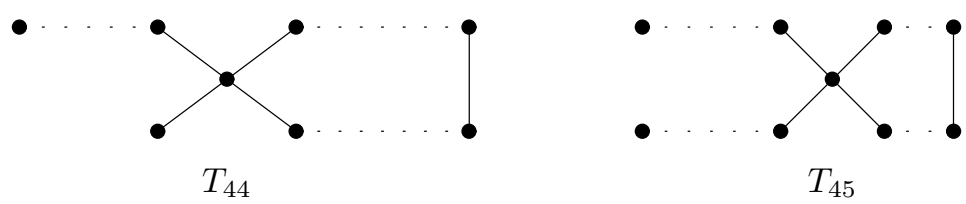

Fig.7

Proof. Let $G_{2}$ be a cospectral to $G_{1}$ with respect to the signless Laplacian matrix. Let $H_{1}$ and $H_{2}$ be the line graphs of $G_{1}$ and $G_{2}$, respectively. By Corollary 2.10, $H_{1}$ and $H_{2}$ are cospectral with respect to the adjacency matrix. If $G_{1}=K_{1,4}$, then $H_{1}=K_{4}$ and so $H_{2}=K_{4}$. Hence $G_{1}$ and $G_{2}$ are isomorphic. Now let $G_{1} \neq K_{1,4}$. By Lemmas 3.2 and 3.5, $0<x_{4} \leq 4$. Hence we have the following cases :

Case1: $x_{4}=4$. By Lemma 3.3 and the fact that $N_{H_{2}}\left(K_{3}\right)=4, H_{2}$ has a subgraph of type $T_{i}$ or $K_{4}$ for $i \in\{42,43\}$ (see Fig.6 ). Since by Lemma 2.12, $H_{2}$ does not have $T_{3}$ as an induced subgraph and the fact that $N_{H_{2}}\left(K_{3}\right)=4, H_{2}$ does not have $T_{42}$ as a subgraph. If $i=43$, then $N_{H_{2}}\left(C_{4}\right) \geq 1$ and by iv) of Lemma 3.2, we have $x_{3}=x_{0}=0$ and $x_{1}=4$. Hence $H_{2}$ contains two path as a component and so $G_{2}$ has more than one bipartite component. Which is a contradiction. Therefor $H_{2}$ has $K_{4}$ as a subgraph. Again by iv) of Lemma 3.2, we have $x_{3}=x_{0}=0$ and $x_{1}=4$. First let $H_{2}$ is a connected graph, then $H_{2}$ is the line graph of a starlike tree with maximum degree 4 . Hence by Theorem 2.5, $H_{1}$ is isomorphic to $H_{2}$ and so by Theorem 2.7, 
$G_{1}$ is isomorphic to $G_{2}$. Now let $H_{2}$ is not a connected graph. Since $x_{3}=x_{0}=0$ and $x_{1}=4$, using the fact that $G_{2}$ has exactly one bipartite component, $H_{2}$ has two components, one of them is path and another is of type $T_{47}$ (see Fig.8).

Case2: $x_{4}=3$. Then by Lemma 3.3 and this fact that $N_{H_{2}}\left(K_{3}\right)=4, H_{2}$ has a subgraph of type $T_{i}$ or $K_{4}$ for $i \in\{39,40,41\}$ (see Fig.6 ). If $i=41$, then $N_{H_{2}}\left(C_{4}\right)>2$ and by Lemma 3.2, we have $x_{3}<0$. Which is impossible. Let $i \in\{39,40\}$. If $x_{0}=1$, then by Lemma 3.2, $x_{1}=x_{3}$ and by Lemma $3.4, x_{1}+2 N=x_{3}$. If $x_{0}=0$ and $H_{2}$ contains exactly one path as a component, then $x_{1}+2 N=x_{3}+2$ and $x_{1}=x_{3}+2$. Hence $N=0$. However $G_{2}$ has more than one bipartite component which is a contradiction. If $H_{2}$ contains no any path as a component, then by Lemma $3.2, x_{1}=x_{3}+2$ and by Lemma $3.4, x_{1}+2 N=x_{3}$. So $N=-1$, which is impossible. So $H_{2}$ has $K_{4}$ as a subgraph. Since $H_{2}$ does not have $K_{1,3}$ as an induced subgraph and $N_{H_{2}}\left(K_{3}\right)=4$, each vertex of degree at least 3 of $H_{2}$ is the vertex of subgraph $K_{4}$ of $H_{2}$. Therefore $x_{3}+x_{4}=4$ and so $x_{3}=1$. On the other hand by Lemma 3.2, $N_{H_{2}}\left(C_{4}\right) \in\{1,2\}$. First let $N_{H_{2}}\left(C_{4}\right)=2$. By Lemma $3.2, y_{4}=4, x_{0}=0$ and $x_{1}=3$ and so $H_{2}$ has 2 components, one of them is a path and another is of type $T_{48}$ (see Fig.8 ). By Lemma 2.11, $N_{H_{2}}(5)=350<N_{H_{1}}(5)=360$, which is not true. Now let $N_{H_{2}}\left(C_{4}\right)=1$. By Lemma $3.2, y_{4}=3, x_{0}=0$ and $x_{1}=3$. First let $H_{2}$ is a connected graph, then it is the line graph of a starlike tree with maximum degree 4 . Hence by Theorem 2.5, $H_{1}$ is isomorphic to $H_{2}$ and so by Theorem 2.7, $G_{1}$ is isomorphic to $G_{2}$. Now let $H_{2}$ is not a connected graph. Since $x_{0}=0$ and $x_{1}=3$, using the fact that $G_{2}$ has exactly one bipartite component, $H_{2}$ has two components, one of them is path and another is of type $T_{48}$.

Case3: Let $x_{4}=2$. Then $H_{2}$ has a subgraph of type $T_{i}$ or $K_{4}$ for $33 \leq i \leq 38$ (see Fig.6 ). Since $x_{4}=2$ and $N_{H_{2}}\left(K_{3}\right)=4$, if $H_{2}$ has $T_{37}$ as a subgraph, then $H_{2}$ has $T_{2}$ as an induced subgraph. By Lemma 2.12, which is impossible.

Let $i=38$, if $x_{0}=1$, then by Lemma 3.2, $x_{1}=x_{3}-2$ and by Lemma 3.4, $x_{1}+2 N=x_{3}$. If $x_{0}=0$ and $H_{2}$ contains exactly one path as a component, then $x_{1}+2 N=x_{3}+2$, $x_{1}=x_{3}$. Hence $N=1$, which is a contradiction to this fact that $G_{2}$ has exactly one bipartite component. If $H_{2}$ contains no path as a component, then $x_{1}+2 N=x_{3}$ and $x_{1}=x_{3}$ so $N=0$. By i) of Lemma 3.2 , it is a contradiction.

Let $i \in\{33,35,36\}$. If $x_{0}=1$, then by Lemma $3.2, x_{1}=x_{3}-2$ and by Lemma 3.4, $x_{1}+2 N=x_{3}-2$. If $x_{0}=0$ and $H_{2}$ contains exactly one path as a component, then $x_{1}+2 N=x_{3}$ and $x_{1}=x_{3}$. Hence $N=0$, by i) of Lemma 3.2, is a contradiction. If $H_{2}$ contains no path as a component, then $x_{1}+2 N=x_{3}-2$ and $x_{1}=x_{3}$ so $N=-1$, which is impossible.

Let $i=34$. If $x_{0}=1$, then by Lemma 3.2, $x_{1}=x_{3}-2$ and by Lemma 3.4, $x_{1}+2 N=x_{3}-2$. So $N=0$. By Lemma 3.2, we have $x_{3}=2 y_{4}-3$. By Lemma 2.11, $N_{H_{2}}(5)=190+20 y_{4}<N_{H_{1}}(5)=240+30 y_{4}$. Which is a contradiction to this fact $H_{1}$ and $H_{2}$ are cospectral with respect to adjacency matrix. If $H_{2}$ contains no path as a component, then $x_{1}+2 N=x_{3}-2$ and $x_{1}=x_{3}$. So $N=-1$, which is impossible. 
If $x_{0}=0$ and $H_{2}$ contains exactly one path as a component, then $x_{1}+2 N=x_{3}$ and $x_{1}=x_{3}$. So $N=0$. By Lemma 3.2, we have $x_{3}=2 y_{4}-2$. By Lemma 2.11, $N_{H_{2}}(5)=200+20 y_{4}<N_{H_{1}}(5)$. Which is a contradiction to this fact $H_{1}$ and $H_{2}$ are cospectral with respect to adjacency matrix. So $H_{2}$ has $K_{4}$ as a subgraph. Since $H_{2}$ does not have $K_{1,3}$ as an induced subgraph and $N_{H_{2}}\left(K_{3}\right)=4$, each vertex of degree at least 3 of $H_{2}$ is the vertex of subgraph $K_{4}$ of $H_{2}$. Therefore $x_{3}+x_{4}=4$ and so $x_{3}=2$. Moreover $H_{2}$ does not have any cycle as a component. So $H_{2}$ has exactly one non-tree component. If $H_{2}$ is a connected graph, then by Lemma 3.2, $x_{1}=2$ and so $N_{H_{2}}\left(C_{4}\right)=1$ and $y_{4}=2$. Therefore $H_{2}$ is a line graph of a starlike graph with maximum degree 4. Using Theorem 2.5, $H_{1}$ and $H_{2}$ are isomorphic. So by Theorem 2.7, $G_{1}$ and $G_{2}$ are isomorphic. If $H_{2}$ is not a connected graph, then by Lemma 3.2, we have $x_{0}+2 N_{H_{2}}\left(C_{4}\right)=2\left(y_{4}-1\right)$. Since $x_{0} \leq 1$, we have $x_{0}=0$ and so $x_{1}=2$ and $N_{H_{2}}\left(C_{4}\right)=y_{4}-1$. Hence $H_{2}$ has 2 components one of them is path and another is of type $T_{46}$. It is easy to see that $N_{H_{2}}\left(C_{4}\right) \leq 2$. If $N_{H_{2}}\left(C_{4}\right)=2$, then $y_{4}=3$ and so $N_{H_{1}}(5)=330>N_{H_{2}}(5)=320$. That is false. Hence $N_{H_{2}}\left(C_{4}\right)=1$ and so $y_{4}=2$. One can successively subdivide certain edges of the $H_{2}$ in an appropriate way, to obtain graph $\tilde{H}$, such that $H_{1}$ can be embedded in $\tilde{H}$ as a proper subgraph. So by Lemma 2.8, $\lambda_{1}\left(H_{2}\right) \geq \lambda_{1}(\tilde{H})$ and by Lemma $2.6, \lambda_{1}(\tilde{H})>\lambda_{1}\left(H_{1}\right)$. Hence $\lambda_{1}\left(H_{2}\right)>\lambda_{1}\left(H_{1}\right)$ which is a contradiction to the fact that $H_{2}$ and $H_{1}$ are cospectral with respect to the adjacency matrix.

Case4: Let $x_{4}=1$. Then $H_{2}$ has a subgraph of type $T_{i}$ or $K_{4}$ for $22 \leq i \leq 32$ (see Fig.6 ). If $H_{2}$ has a subgraph of type $K_{4}$, then by Lemma 3.2, $H_{2}$ is a line graph of a starlike graph with maximum degree 4. Using Theorem 2.5, $H_{1}$ and $H_{2}$ are isomorphic. So by Theorem 2.7, $G_{1}$ and $G_{2}$ are isomorphic. Now let $H_{2}$ has a subgraph of type $T_{i}$ for $22 \leq i \leq 32$.

Let $i \in\{22,27,28\}$. If $x_{0}=1$, then by Lemma $3.2, x_{1}=x_{3}-4$ and by Lemma 3.4 , $x_{1}+2 N=x_{3}-4$. If $x_{0}=0$ and $H_{2}$ contains exactly one path as a component, then $x_{1}+2 N=x_{3}-2$ and $x_{1}=x_{3}-2$. Any way, $N=0$. So $G_{2}$ has more than one bipartite component, which is impossible. If $\mathrm{H}_{2}$ contains no path as a component, then $x_{1}+2 N=x_{3}-4$ and $x_{1}=x_{3}-2$. So $N=-1$, which is impossible.

Let $i=23$. If $x_{0}=1$, then by Lemma 3.2, $x_{1}=x_{3}-4$ and by Lemma 3.4, $x_{1}+2 N=$ $x_{3}-4$. Also if $x_{0}=0$ and $H_{2}$ contains exactly one path as a component, then $x_{1}+2 N=x_{3}-2$ and $x_{1}=x_{3}-2$. Any way we have $N=0$. Thus $N_{H_{2}}\left(C_{4}\right)=1$. By Lemma 3.2, we have $x_{3}=2 y_{4}$ or $x_{3}=2 y_{4}+1$. By Lemma 2.11, $N_{H_{2}}(5)=180+20 y_{4}$ or $N_{H_{2}}(5)=190+20 y_{4}$. However $N_{H_{2}}(5)<N_{H_{1}}(5)$, that is impossible. If $H_{2}$ contains no path as a component, then by Lemma 3.2, $x_{1}=x_{3}-2$ and by Lemma $3.4, x_{1}+2 N=x_{3}-4$. So $N=-1$, which is impossible.

Let $i=30$. If $x_{0}=1$, then by Lemma 3.2, $x_{1}=x_{3}-4$ and by Lemma 3.4, $x_{1}+2 N=x_{3}-2$. Also if $x_{0}=0$ and $H_{2}$ contains exactly one path as a component, then $x_{1}+2 N=x_{3}$ and $x_{1}=x_{3}-2$. Any way we have $N=1$. Thus $N_{H_{2}}\left(C_{4}\right)=$ 1 or 2 . If $N_{H_{2}}\left(C_{4}\right)=1$, then by Lemma 3.2 , we have $x_{3}=2 y_{4}$ or $x_{3}=2 y_{4}+1$. 
By Lemma 2.11, $N_{H_{2}}(5)=180+20 y_{4}$ or $N_{H_{2}}(5)=190+20 y_{4}$. If $N_{H_{2}}\left(C_{4}\right)=2$, then $x_{3}=2 y_{4}-2$ or $x_{3}=2 y_{4}-1$. By Lemma 2.11, $N_{H_{2}}(5)=160+20 y_{4}$ or $N_{H_{2}}(5)=170+20 y_{4}$. However $N_{H_{2}}(5)<N_{H_{1}}(5)$, this is a contradiction to this fact $H_{1}$ and $H_{2}$ are cospectral with respect to adjacency matrix. If $H_{2}$ contains no any path as a component, then by Lemma 3.2, $x_{1}=x_{3}-2$ and by Lemma $3.4, x_{1}+2 N=x_{3}-2$. So $N=0$. Hence by Lemma $3.2, x_{3}=2 y_{4}+1$. So $N_{H_{2}}(5)=190+20 y_{4}<N_{H_{1}}(5)$. This is impossible.

Let $i \in\{31,32\}$. If $x_{0}=1$, then by Lemma 3.2, $x_{1}=x_{3}-4$ and by Lemma 3.4, $x_{1}+2 N=x_{3}-2$. If $x_{0}=0$ and $H_{2}$ contains exactly one path as a component, then $x_{1}+2 N=x_{3}$ and $x_{1}=x_{3}-2$. So $N=1$. However $G_{2}$ has more than one bipartite component and this is a contradiction. If $H_{2}$ contains no path as a component, then $x_{1}+2 N=x_{3}-2$ and $x_{1}=x_{3}-2$. So $N=0$, which is a contradiction to this fact that $G_{2}$ has exactly one bipartite component.

Let $24 \leq i \leq 26$. If $x_{0}=1$, then by Lemma $3.2, x_{1}=x_{3}-4$ and by Lemma 3.4, $x_{1}+2 N=x_{3}-4$. If $x_{0}=0$ and $H_{2}$ contains exactly one path as a component, then $x_{1}+2 N=x_{3}-2$ and $x_{1}=x_{3}-2$. So $N=0$. By Lemma 3.2, for $i=24$, we have $x_{3}=2 y_{4}-2$ or $x_{3}=2 y_{4}-1$. By Lemma $2.11, N_{H_{2}}(5)=180+20 y_{4}$ or $N_{H_{2}}(5)=$ $190+20 y_{4}$. By Lemma 3.2, for $i=25$, we have $x_{3}=2 y_{4}$ or $x_{3}=2 y_{4}+1$. By Lemma $2.11, N_{H_{2}}(5)=180+20 y_{4}$ or $N_{H_{2}}(5)=190+20 y_{4}$. However $N_{H_{2}}(5)<N_{H_{1}}(5)$, that is not true. Now if $x_{0}=0$ and $H_{2}$ contains no path as a component, then by Lemma 3.2, $x_{1}=x_{3}-2$ and by Lemma $3.4, x_{1}+2 N=x_{3}-4$. So $N=-1$, which is impossible. Since $x_{4}=1$ and $N_{H_{2}}\left(K_{3}\right)=4$, if $H_{2}$ has $T_{26}$ as a subgraph, then $H_{2}$ has $T_{2}$ as an induced subgraph. By Lemma 2.12, which is impossible.

Let $i=29$. If $x_{0}=1$, then by Lemma 3.2, $x_{1}=x_{3}-4$ and by Lemma 3.4, $x_{1}+2 N=x_{3}-2$. If $x_{0}=0$ and $H_{2}$ contains exactly one path as a component, then $x_{1}+2 N=x_{3}$ and $x_{1}=x_{3}-2$. Hence $N=1$. Thus $N_{H_{2}}\left(C_{4}\right)=1$ or 2 . If $N_{H_{2}}\left(C_{4}\right)=1$, then by Lemma 3.2 , we have $x_{3}=2 y_{4}$ or $x_{3}=2 y_{4}+1$. By Lemma $2.11, N_{H_{2}}(5)=180+20 y_{4}$ or $N_{H_{2}}(5)=190+20 y_{4}$. If $N_{H_{2}}\left(C_{4}\right)=2$, then by Lemma 3.2, we have $x_{3}=2 y_{4}-2$ or $x_{3}=2 y_{4}-1$. By Lemma 2.11, $N_{H_{2}}(5)=160+20 y_{4}$ or $N_{H_{2}}(5)=170+20 y_{4}$, a contradiction. If $x_{0}=0$ and $H_{2}$ contains no path as a component, then by Lemma 3.2, $x_{1}=x_{3}-2$ and by Lemma $3.4, x_{1}+2 N=x_{3}-2$. So $N=0$. Thus by Lemma $3.2, x_{3}=2 y_{4}+1$. By Lemma $2.11, N_{H_{2}}(5)=190+20 y_{4}<N_{H_{1}}(5)$. This is impossible. 

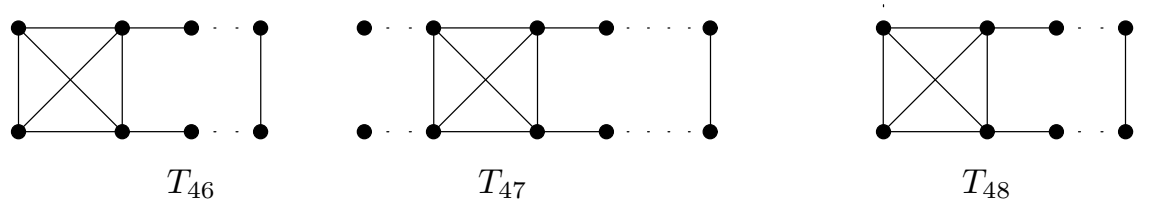

Fig.8

Let $N_{G}(H)$ be the number of subgraphs of a graph $G$ which are isomorphic to $H$ and let $N_{G}(i)$ be the number of closed walks of length $i$ in $G$. Let $N_{H}^{\prime}(i)$ be the number of closed walks of length $i$ of $H$ which contains all edges and let $S_{i}(G)$ be the set of all connected graphs such $H$ with $N_{H}^{\prime}(i) \neq 0$ where $G$ has at least one subgraph isomorphic to $H$. Then:

$$
N_{G}(i)=\sum_{H \in S_{i}(G)} N_{G}(H) N_{H}^{\prime}(i) .
$$

Theorem 3.7. Let $G=S(a, b, c, d)$ where $d \geq c \geq b \geq a \geq 1$. Then $G$ is determined by its signless Laplacian spectrum.

Proof. Let $G_{1}=G$ and let $G_{2}$ be cospectral to $G_{1}$ with respect to the signless Laplacian matrix. If $G_{2}$ is not isomorphic to $G_{1}$, then by using Theorem 3.6, we have the following cases:

Case1: Let $a=1, b>1$ and let $G_{2}=A$ (see Fig.9). If $\bar{b} \geq b$, then we can subdivide certain edges of the cycle $C_{l}$ of $L\left(G_{2}\right)$ in an appropriate way, to obtain graph $\tilde{H}$, such that $L\left(G_{1}\right)$ can be embedded in $\tilde{H}$ as a proper subgraph. So by Lemma 2.8, $\lambda_{1}\left(L\left(G_{2}\right)\right) \geq \lambda_{1}(\tilde{H})$ and by Lemma 2.6, $\lambda_{1}(\tilde{H})>\lambda_{1}\left(L\left(G_{1}\right)\right)$. Hence $\lambda_{1}\left(L\left(G_{2}\right)\right)>\lambda_{1}\left(L\left(G_{1}\right)\right)$ which contradicts to the fact that $L\left(G_{2}\right)$ and $L\left(G_{1}\right)$ are cospectral with respect to the adjacency matrix. So $\bar{b}<b$. If $\bar{b} \geq(l-1) / 2$, then $S_{l}\left(L\left(G_{2}\right)\right)=S_{l}\left(L\left(G_{1}\right)\right) \cup\left\{C_{l}\right\}$ and for each $K \in S_{l}\left(L\left(G_{1}\right)\right), N_{L\left(G_{2}\right)}(K) \geq N_{L\left(G_{1}\right)}(K)$. So by the equation (3.3), $N_{L\left(G_{2}\right)}(l)>N_{L\left(G_{1}\right)}(l)$, contradicting to the fact that $L\left(G_{2}\right)$ and $L\left(G_{1}\right)$ have the same number of closed walks of any length. If $\bar{b}<(l-3) / 2$, then $S_{(2 \bar{b}+3)}\left(L\left(G_{2}\right)\right)=S_{(2 \bar{b}+3)}\left(L\left(G_{1}\right)\right), N_{L\left(G_{1}\right)}(K(1,1, \bar{b}+1))>N_{L\left(G_{2}\right)}(K(1,1, \bar{b}+1))$ and $N_{L\left(G_{1}\right)}(K)=N_{L\left(G_{2}\right)}(K)$ for each $K \neq K(1,1, \bar{b}+1)$ in $S_{(2 \bar{b}+3)}\left(L\left(G_{2}\right)\right)$. Hence by the equation (3.3), we have $N_{L\left(G_{1}\right)}(2 \bar{b}+3)>N_{L\left(G_{2}\right)}(2 \bar{b}+3)$, which is again a contradiction. Hence $\bar{b} \in\{(l-3) / 2,(l-2) / 2\}$. On the other hand $G_{1}$ and $G_{2}$ have the same number of vertices and so $l>c+d \geq 2 b \geq 2 \bar{b}+2$. Therefore $\bar{b}=(l-3) / 2$ and so $G_{2}$ is not a bipartite graph, contradicting to the fact that $G_{1}$ is bipartite.

Case2: Let $a>1$ and let $G_{2}$ has two components, one of them is path and another is 
$B$ (see Fig.9) where $\bar{a} \leq \bar{b}$. Since $G_{2}$ has exactly one bipartite component, $l$ is an odd number. If $\bar{a}>a$, then by easy task we can see that $N_{L\left(G_{2}\right)}(2 a+3)>N_{L\left(G_{1}\right)}(2 a+3)$, contradicting to the fact that $L\left(G_{2}\right)$ and $L\left(G_{1}\right)$ have the same number of closed walks of any length. If $\bar{a}=a$, then as a similar to case1, we have $\bar{b}=(l-3) / 2<b$. Since for each natural $x$ we have $N_{K(1,1, x)}^{\prime}(2 x+1)=N_{C_{(2 x+1)}}^{\prime}(2 x+1)=4 x+2$, it is easy to see that $N_{L\left(G_{2}\right)}(l)<N_{L\left(G_{1}\right)}(l)$, that is impossible. If $\bar{a}<a$, then again as a similar to case1, we have $\bar{a}=(l-3) / 2$. Again we can see that $N_{L\left(G_{2}\right)}(l)<N_{L\left(G_{1}\right)}(l)$, which is impossible.

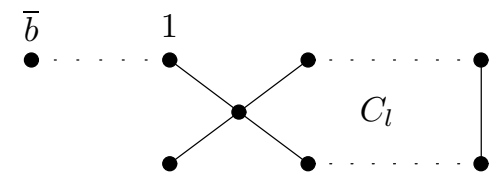

$A$

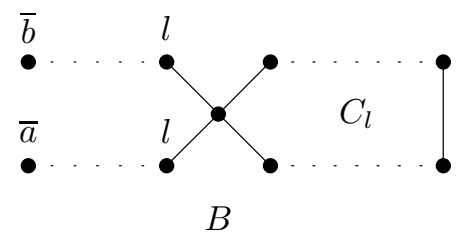

$B$

Fig.9

Acknowledgment. The authors are very grateful to the referee for his/her useful comments.

REFERENCES

[1] J. A. Bondy and U. S. R. Murty. Graph Theory with Applications. The MacMillan Press Ltd, 1976.

[2] D. M. Cvetkovic, M. Doob, and H. Sachs. Spectra of Graphs. Third edition, Johann Abrosius Barth Verlag, 1995.

[3] A. J. Hoffman and J. H. Smith. On the spectral radii of topologically equivalent graphs. In Recent Advances in Graph Theory, Academia Praha, M. Fiedler, Editor, (1975) pp. 273-281.

[4] M. Lepović. Some results on starlike trees and sunlike graphs. Journal of Applied Mathematics and Computing, 11:109-123, 2003.

[5] G. R. Omidi. On a signless Laplacian spectral characterization of T-shape trees. Linear Algebra and its Applications, 431:1607-1615, 2009.

[6] G. R. Omidi and K. Tajbakhsh. Starlike trees are determined by their Laplacian spectrum. Linear Algebra and its Applications, 422:654-658, 2007.

[7] X. L. Shen, Y. P. Hou, and Y. P. Zhang. Graph $Z_{n}$ and some graphs related to $Z_{n}$ are determined by their spectrum. Linear Algebra and its Applications, 404: 58-68, 2005.

[8] E. R. van Dam and W. H. Haemers. Which graphs are determined by their spectrum? Linear Algebra and its Applications, 373:241-272, 2003.

[9] W. Wang and C. X. Xu. On the spectral charactrization of T-shape trees. Linear Algebra and its Applications, 414:492-501, 2006.

[10] W. Wang and C. X. Xu. Note: The T-shape tree is determined by its Laplacian spectrum. Linear Algebra and its Applications, 419:78-81, 2006. 